\title{
The AMBRE Project: $r$-process element abundances in the Milky Way thin and thick discs
}

\section{Guillaume Guiglion $^{1}$, Patrick de Laverny ${ }^{2}$, Alejandra Recio-Blanco ${ }^{2}$ and C. Clare Worley ${ }^{3}$}

${ }^{1}$ Leibniz-Institut für Astrophysik Potsdam (AIP) An der Sternwarte 16, 14482 Potsdam email: gguiglion@aip.de

${ }^{2}$ Université Côte d'Azur, Observatoire de la Côte d'Azur, CNRS, Laboratoire Lagrange, France

${ }^{3}$ Institute of Astronomy, University of Cambridge, Madingley Rise, Cambridge CB3 0HA, UK

\begin{abstract}
Chemical evolution of $r$-process elements in the Milky Way disc is still a matter of debate. We took advantage of high resolution HARPS spectra from the ESO archive in order to derive precise chemical abundances of $3 r$-process elements $\mathrm{Eu}, \mathrm{Dy} \& \mathrm{Gd}$ for a sample of 4355 FGK Milky Way stars. The chemical analysis has been performed thanks to the automatic optimization pipeline GAUGUIN. Based on the $[\alpha / \mathrm{Fe}]$ ratio, we chemically characterized the thin and the thick discs, and present here results of these $3 r$-process element abundances in both discs. We found an unexpected Gadolinium and Dysprosium enrichment in the thick disc stars compared to Europium, while these three elements track well each other in the thin disc.
\end{abstract}

Keywords. stars: abundances. techniques: spectroscopic. Galaxy: abundances. Galaxy: stellar content

\section{Motivations, observations and chemical abundances}

The synthesis of heavier elements than Iron is suppose to take place in stellar interiors, via capture of neutrons. Two main families of such elements are known, from slow-neutron capture $(s-)$ or rapid- neutron capture $(r-)$ compared to the timescale for $\beta^{-}$decay. In this study we will focus on $3 r$-process elements: Eu, Gd and Dy. These elements are supposed to be formed via neutrino-induced winds from SN II (Woosley et al. 1994), merging of neutron stars (Freiburghaus 1999) or polar jets from SN II via pure MHD explosion (Nishimura et al. 2006), but current yields suffer from large uncertainties. On the observational side, there is a current lack of massive homogeneous catalog of $r$ process abundances in order to put constraints on their chemical evolution in the Milky Way. In this study, we took advantage of the ESO spectroscopic archive, focusing of the high resolution $(R \sim 110000)$ HARPS data. Our working sample is composed of 4355 individual stars with high quality atmospheric parameters $\left(T_{\text {eff }}, \log (g),[\mathrm{Fe} / \mathrm{H}]\right.$, $[\alpha / \mathrm{Fe}])$ derived by the AMBRE Project (De Pascale et al. 2014). We built a line-list based on VALD3 (Kupka et al. 1999) atomic blends, molecular blends and most recent laboratory and theoretical $\log g f$ measurements for the Eu (Lawler et al. 2001), Gd (Den Hartog et al. 2006) and Dy (Wickliffe et al. 2000). We used the optimization pipeline GAUGUIN (Guiglion et al. 2016) is order to derive the individual chemical abundances. We selected the best abundances for stars with $T_{\text {eff }}>4500 \mathrm{~K}$ and $S / N>50$, resulting in a sub-sample of 1672 stars. Typical errors are 0.12 dex for Eu and Gd and 0.11 dex for Dy. 

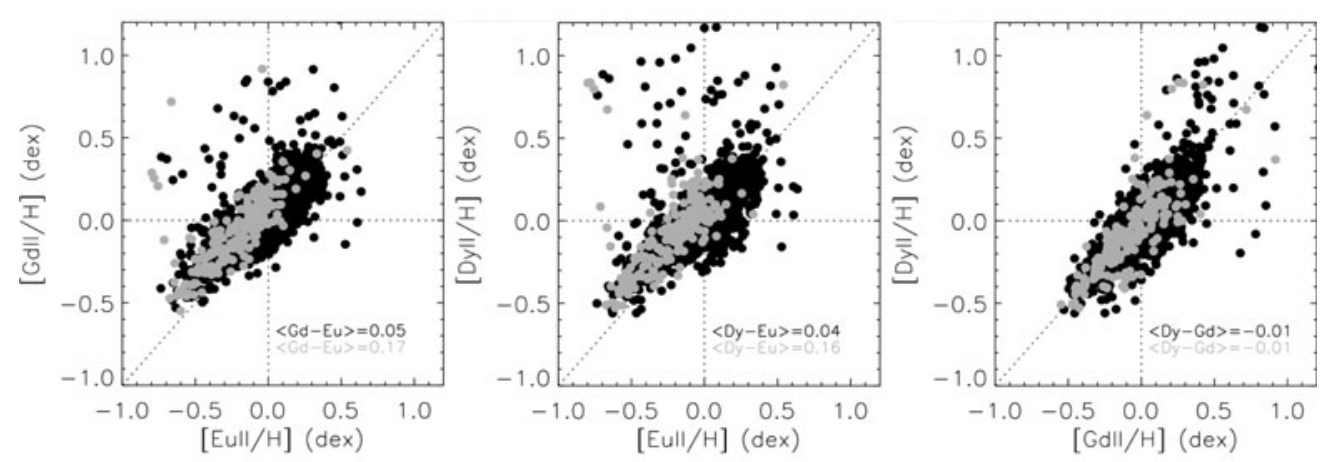

Figure 1. Gd v.s. Eu, Dy v.s. Eu and Dy v.s. Gd in the thin disc (black, filled) and the thick disc (grey, filled). Average differences are noted.

\section{Looking for $r$-process enhancement in the thin \& thick discs}

We first chemically characterized the thin and the thick discs, taking advantage of the $[\alpha / \mathrm{Fe}]$ ratio, following the same procedure as in (Guiglion et al. 2016). We identified 1469 stars in the thin disc, 182 in the thick disc. We showed that the [Eu,Gd,Dy/Fe] ratios decrease with $[\mathrm{M} / \mathrm{H}]$, following typical $\alpha$-element trend. The thick discs shows higher $[\mathrm{Eu}, \mathrm{Gd}, \mathrm{Dy} / \mathrm{Fe}]$ ratios with respect to the thin disc. It is the first time that the $\mathrm{Gd}$ and Dy contents are characterized in the thin and the thick disc stars. The Eu pattern is in agreement with recent observations (ex Battistini et al. 2016) and chemical evolution model (Travaglio et al. 2009). We note that Gd and Dy show very similar patterns, in both discs, suggesting a common chemical evolution. In addition, we observe that $[\mathrm{Eu}, \mathrm{Gd}, \mathrm{Dy} / \mathrm{Fe}]$ ratios are strongly correlated to $[\alpha / \mathrm{Fe}]$. Indeed, Eu, Gd \& Dy increase as a function of $[\alpha / \mathrm{Fe}]$ in both discs, suggesting a higher chemical enrichment in r-process at early time.

A presented in Figure 1, we first observe that in the thin disc, Eu, Gd and Dy track well each other, without showing significant offset. On the other hand, we clearly see in the thick disc that Gd and Dy are enhanced compared to Eu, respectively of 0.17 and 0.16 dex (left and middle panel), while Gd and Dy track each other well (right panel). We think that the offset is not related to any line-list calibration issues because no significant offsets is observed in the thin disc stars. It is unexpected that Gd and Dy show an enrichment compared to $\mathrm{Eu}$ in the thick disc, because these three elements should originate from the same sources and sites of production. Up to now, no galactic chemical evolution model is able to explain such enrichment for these two $r$-process elements. Further investigations, especially thanks to the stellar ages, will be needed to understand such observations.

\section{References}

Battistini, C. \& Bensby, T., 2016, A\& 4 , 586, A49

Den Hartog, E. A., Lawler, J. E., Sneden, C., \& Cowan, J. J., 2006, ApJS, 167, 292.

De Pascale, M., Worley, C. C., de Laverny, P., et al. 2014, A\&A, 570, A68

Freiburghaus, C., Rosswog, S., \& Thielemann, F.-K. 1999, ApJ, 525, L121

Guiglion, G, de Laverny, P., Recio-Blanco, A., Worley, C. C., et al. A $\& A, 2016,595$, A18

Kupka, F., Piskunov, N., Ryabchikova, T. A., Stempels, H. C., et al. A\&\&A, 1999, 138, 119

Lawler, J. E., Wickliffe, M. E., den Hartog, E. A., \& Sneden, C. 2001, ApJ, 563, 1075.

Nishimura, S., Kotake, K., Hashimoto, M., et al. 2006, ApJ, 642, 410

Travaglio, C., Galli, D., Gallino, R., et al. 1999, ApJ, 521, 69

Wickliffe, M. E., Lawler, J. E., \& Nave, G., 2000, J. Quant. Spec. Radiat. Transf., 2000, 66, 363

Woosley, S. E., Wilson, J. R., Mathews, G. J., Hoffman, R. D., et al. 1994, ApJ, 433, 229 